\title{
TOWARDS A DIGITAL TWIN OF A STORAGE TANK USING LASER SCAN DATA
}

\author{
L. Truong-Hong ${ }^{1, *}$, N. Nguyen ${ }^{2}$, R. Lindenbergh ${ }^{1}$, P. Fisk ${ }^{3}$, T. Huynh ${ }^{2}$ \\ ${ }^{1}$ Dept. of Geoscience \& Remote Sensing, Delft University of Technology, Delft, The Netherland, - (1.truong; \\ r.c.lindenbergh)@tudelft.nl \\ ${ }^{2}$ Faculty of Civil Engineering, Ho Chi Minh city University of Technology, Ho Chi Minh City, Viet Nam, - \\ (1612357; huynhngocthi)@hcmut.edu.vn \\ ${ }^{3}$ Ironhide Inspection Inc., Calgary, Canada - paul@ironhideinspection.ca
}

KEY WORDS: Point clouds; Storage tank; Segmentation; Deformation; Finite element analysis; Digital twin

\begin{abstract}
:
This paper proposes a methodology to automatically extract components of an oil storage tank from terrestrial laser scanning (TLS) point clouds, and subsequently to create a three-dimensional (3D) solid model of the tank for numerical simulation. The proposed method is integrated into a smart analysis layer of a digital twin platform consisting of three main layers: (1) smart analysis, (2) data storage, and (3) visualisation and user interaction. In this proposed method, primary components of the tank were automatically extracted in a consecutive order from a shell wall to roof and floor. Voxel-based RANSAC is employed to extract voxels containing point clouds of the shell wall, while a valley-peak-valley pattern based on kernel density estimation is implemented to remove outlier points within voxels representing to the shell wall and re-extract data points within voxels adjoined to the shell wall. Moreover, octreebased region growing is employed to extract a roof and floor from remaining point clouds. An experimental showed that the proposed framework successfully extracted all primary components of the tank and created a 3D solid model of the tank automatically. Resulting point clouds of the shell wall were directly used for estimating deformation and a 3D solid model was imported into finite element analysis (FEA) software to assess the tank in terms of stress-strain. The demonstration shows that TLS point clouds can play an important role in developing the digital twin of the oil storage tank.
\end{abstract}

\section{INTRODUCTION}

Laser scanning, also known as Light Detection and Ranging (LiDAR), has been used to capture three-dimensional (3D) topographic data of visible surfaces of objects quickly and accurately. Particularly, a terrestrial laser scanner (TLS) captures great details of the surfaces with millimetre accuracy, which are to be widely used for creating three-dimensional (3D) geometric model, and structural engineering, for example structural inspection (Truong-Hong et al., 2021), deformation modelling (Truong-Hong and Lindenbergh, 2019), and finite element mesh (Kassotakis et al., 2020).

In measuring deformation of a tank shell, Pukanská et al. (2014) manually extract data points of the tank shell and used point clouds of a top and bottom sections with the section thickness of $0.1 \mathrm{~m}$ to fit a cylinder representing to the shell. Huadong et al. (2017) sliced point cloud of the shell wall based on known elevations of courses, and a least square method is used to fit the cylinder for the points of each layer. In attempt to automatically extract the points of the shell, Truong-Hong et al. (2020) used voxel-based RANSAC to extract the shell wall for a TLS point cloud capturing from exterior. Moreover, to assess the tank in terms of structural analysis, Wu et al. (2020) used an as-design model in finite element analysis (FEA) to simulate the temperature distribution due to fire. However, the use of the asdesign model in FEA can affect to results because there is discrepancy between the as-design and as-is models (TruongHong and Laefer, 2013).

As a large amount of crude oil stores in oil storage tanks, which can be up to ten thousand of cubic, any accident occurred can trigger catastrophic consequences for both property and environment. As such, inspection and monitoring radically change of the tank would be kept an important role to prevent any risk. A digital twin would be a great platform for engineers able to keep track of the tank real time quantities through deformations, stress and strain. However, converting a physical model to a digital one of the tank is still manual work with high cost and time consuming. Thus, this paper presents an overall framework of a digital twin for oil storage tanks. This study mainly focuses on extracting primary components (a shell wall, roof, and floor) of the tank from a TLS point cloud automatically. Subsequently, point clouds of these components are used to estimate deformation though their point clouds, and automatically create a 3D solid model directly importing into FEA to assess the tank via stress-strain.

\section{PROPOSED METHOD}

A goal of the study is to develop a digital twin framework of oil storage tanks, which consists of two main parts: Part 1 - tank component extraction and Part 2 - a digital twin (Figure 1). Part 1 focusses on extracting TLS point clouds of components of the, in which primary components (a shell wall, roof, and floor) are objective, while other components (e.g., columns, girders, and rafters) are parts of future work. Part II proposes a digital twin framework consisting of three layers: (1) Smart analysis, (2) Data Storage, and (3) Visualisation and User Interaction. In the digital twin platform, this paper focusses on the smart analysis layer to explore ability to use point clouds for 3D geometric model reconstruction, deformation analysis and FEA in an automated manner.

For Part I, a point cloud $\boldsymbol{P}=\left(p_{i}=\left(p_{i .} x, p_{i} . y, p_{i .} z\right) \in R^{3}\right)$ of a tank (Figure 2a) is decomposed into voxels $\left(\boldsymbol{V}=\left(v_{i}\right), i=\left[1, N_{v}\right]\right)$ by using voxel grid with a predefined voxel size ( $\left.v_{0}\right)$ (Step 1.1) (Figure 2b). Subsequently, a voxel is classified as "full" $\left(\boldsymbol{V}_{f}\right)$, if the voxel occupies the number of points larger than a predefined minimum number of points $\left(v_{\text {min_pts }}=10\right)$; otherwise, it classified as "empty" $\left(\boldsymbol{V}_{e}\right)$. In Step 1.2, points $p_{i} \in v_{i} \in \boldsymbol{V}_{f}$ (full voxels) are

\footnotetext{
* Corresponding author
} 
assumed to represent a plane, as such principal component analysis (PCA) (Hoppe et al., 1992) is employed to estimate a local plane describes as $v_{i . s}=\left(v_{i . p}, v_{i . n}\right)$, where $v_{i . p}$ ois a centroid of the points $p_{i}$, and $v_{i} . n$ is the normal vector of a fitting plan. Next, the voxels $\boldsymbol{V}_{f}$ are respectively classified as vertical $\left(\boldsymbol{V}_{v}\right)$ and non-vertical voxels $\boldsymbol{V}_{h}$ (Equation 1), which is based on deviation between the normal vector of the voxel and an unit vector of the axis oz (Figure 2c).

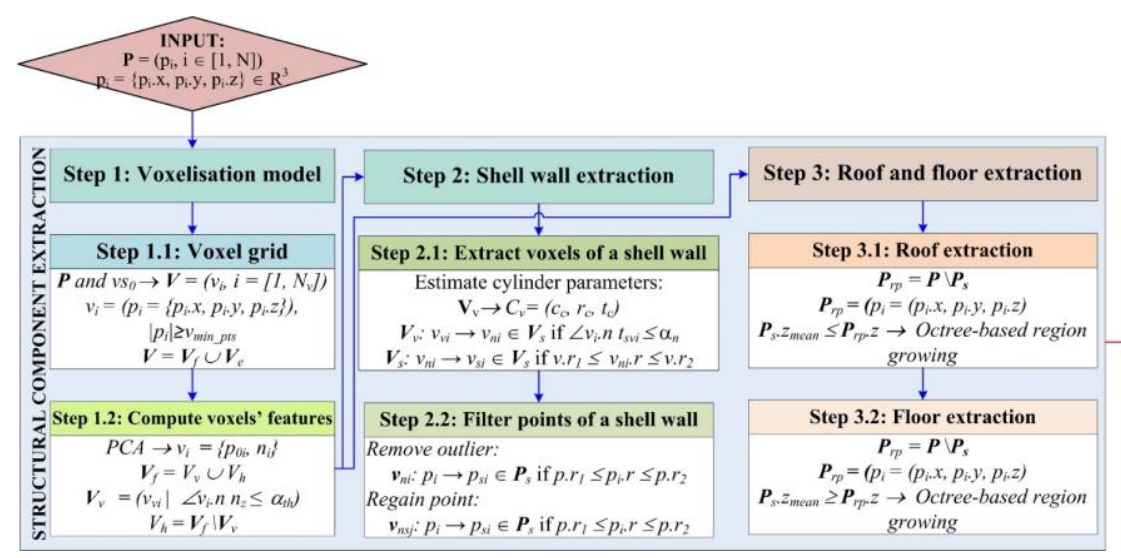

$$
\boldsymbol{V}_{f:} v_{i} \rightarrow v_{v i} \in \boldsymbol{V}_{v} \text { if } \angle v_{i} . n n_{z} \leq \alpha_{t h} ; \boldsymbol{V}_{h}=\boldsymbol{V}_{f} \backslash \boldsymbol{V}
$$

where $\quad n_{z}=$ a unit vector of $o z$ axis

$\alpha_{t h}=$ the predefined angle criterion to determine the voxels contain points of vertical surfaces

Figure 1. A framework to create a digital twin of a storage tank

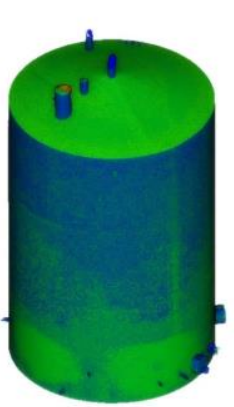

a)

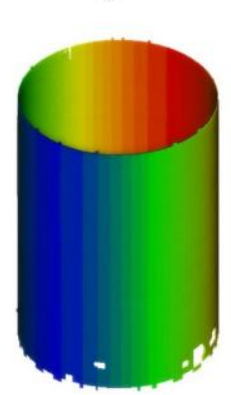

f)

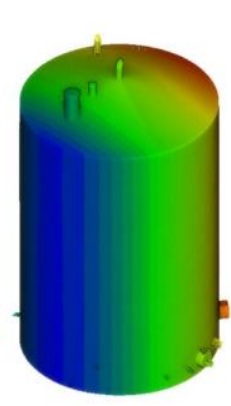

b)
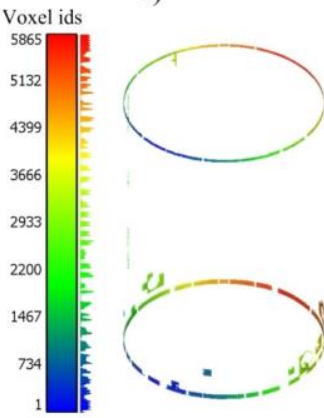

g)

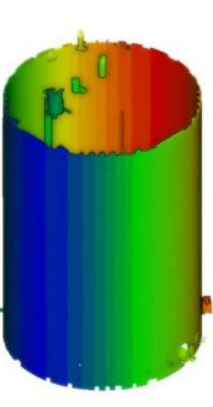

c)
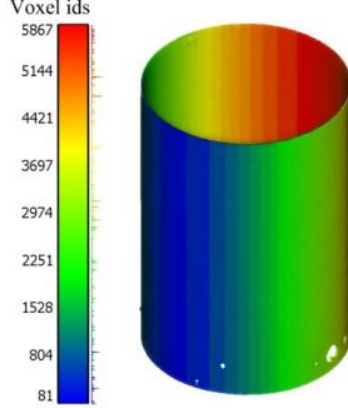

h)

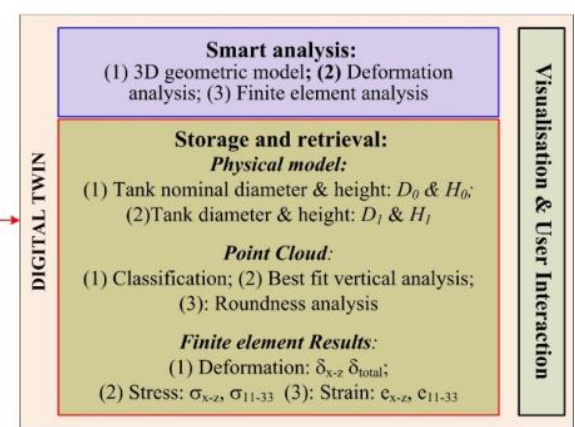

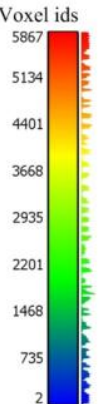

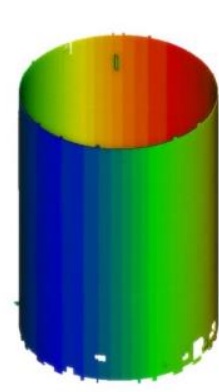

d)

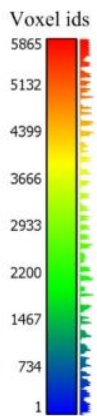

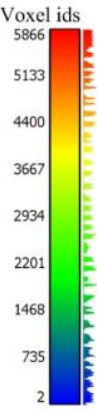

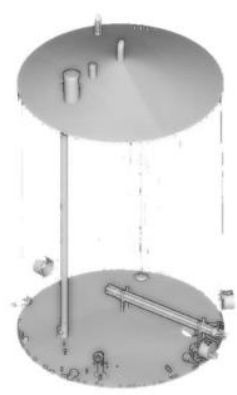

i)

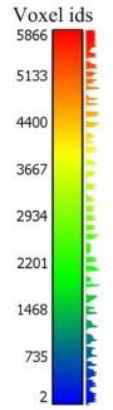

e)

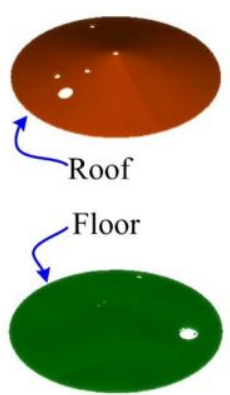

j)

Figure 2. Extracting a shell wall, roof, and floor of a storage tank from TLS point clouds a) Point clouds of a tank, b) a voxel grid model, c) point clouds within vertical voxels $\left(\boldsymbol{V}_{v}\right)$, d) point clouds after applying normal filter $\left(\boldsymbol{V}_{v} \rightarrow \boldsymbol{V}_{s}\right)$, e) point clouds after applying radius filter $\left(\boldsymbol{V}_{s} \rightarrow \boldsymbol{V}_{s}\right)$, f) resulting point clouds of the shell wall after removing outlier points within $v_{s i}$, g) resulting point clouds of the shell wall obtaining from $v_{n s j}$, h) final point clouds $\left(\boldsymbol{P}_{s}\right)$ of the shell wall, i) remaining point clouds $\left.\left(\boldsymbol{P}_{r p}\right), \mathrm{j}\right)$ resulting point clouds of a roof $\left(\boldsymbol{P}_{r}\right)$ and floor $\left(\boldsymbol{P}_{f}\right)$

Step 2 are extracted data points of the tank shell in two sub-steps. In Step 2.1, RANSAC based voxels proposed by Truong-Hong et al. (2020) was employed to estimate the cylinder representing the shell wall from $\boldsymbol{V}_{v}$, in which the cylinder $\left(\mathrm{C}_{\mathrm{v}}\right)$ parameters consist of a center $c_{c}=\left(x_{c}, y_{c}, z_{c}\right)$, a radius $r_{c}$, a directional vector of an axis $t_{c}=\left(t_{x}, t_{y}, t_{z}\right)$. Next, two consecutive filtering steps called normal and radius filters are used to extract the voxels occupied the points of the shell (Equation 2 and 3) (Figure 2d and 2e). The first filter is based the principle is that the point/voxel on the cylinder have a normal perpendicular to the cylinder surface, while the second filter is based on that the voxels on the cylinder surface have nearly the same radius.

$$
\boldsymbol{V}_{v}: v_{v i} \rightarrow v_{n i} \in \boldsymbol{V}_{s} \text { if } \angle v_{i} . n t_{s v i} \leq \alpha_{n}
$$


where $\quad t_{s v i}=$ the vector connected the voxel center $\left(v_{i} . p_{0}\right)$ and its projection on the cylinder axis defined by a center $c_{c}$ and $t_{c}$ $\alpha_{n}=$ an angle criterion for a deviation between the normal of voxels and a normal vector of the cylinder

$$
\boldsymbol{V}_{s}: v_{n i} \rightarrow v_{s i} \in \boldsymbol{V}_{s} \text { if } v_{.} r_{1} \leq v_{n i .} \leq v . r_{2}
$$

where $\quad v_{n i} . r=$ the distance from $v_{n i} . p_{0}$ to the cylinder axis

$v . r_{l}$ and $v . r_{2}=a$ lower and upper bound of the largest peak of KDE generated from $v_{n i} . r$, which is determined by using valley-peak-valley pattern (Truong-Hong and Lindenbergh, 2021).

As $v_{s i}$ may contain points of components (e.g., a roof or pipes) attached to the shell wall, and points of the shell wall may locate with other voxels $\left(v_{n s j} \in \boldsymbol{V} \boldsymbol{V}_{s}\right)$ that are not recognized as $v_{s i}$, Step 2.2 filters those points in two sub-steps. First, for each $v_{s i}$, a radial coordinate $p_{i . r}$, the distance from the point to the cylinder axis, is computed, and the point $p_{i} \in v_{s i}$ belongs to the shell wall points $\left(\boldsymbol{P}_{s}\right)$ (Figure 2f), if it satisfies Equation 4.

$$
\boldsymbol{v}_{n i:} p_{i} \rightarrow p_{s i} \in \boldsymbol{P}_{s} \text { if } p . r_{1} \leq p_{i . r} \leq p . r_{2}
$$

where $\quad p . r_{1}$ and $p . r_{2}=$ a lower and upper bound of the largest peak of KDE generated from $p_{i . r}$

Second, to extract points of the shell wall located within other voxels $v_{n s j}$, the algorithm starts from the voxel $v_{n s j}$ to search adjoined voxels as $v_{s i} \in \boldsymbol{V}_{s}$, and $p_{i} \in v_{n s j}$ is considered as the point of the shell wall points (Figure 2g), if $p_{i . r}$ satisfies Equation 4, in which the bounds $\left[p . r_{1}, p . r_{2}\right]$ are determined KDE generated from $p_{i .} r \in v_{s i}$. Notably, if the $p_{i} \in v_{n s j}$ is labelled as $\boldsymbol{P} s$, the voxel $v_{n s j}$ is labelled as the shell wall voxels $\left(\boldsymbol{V}_{s}\right)$. Resulting final points of the shell wall are shown in Figure $2 \mathrm{~h}$.

In Step 3, after labelling the point $p_{i}$ as the shell wall $\left(\mathbf{P}_{\mathbf{s}}\right)$, remaining points $\boldsymbol{P}_{r p}=\boldsymbol{P} \backslash \boldsymbol{P}_{s}$ where $\boldsymbol{P}_{\mathrm{s}}=\left(p_{i}\right)$ are used to extract the floor and roof (Figure 2i). Notably, the $p_{i} \in \boldsymbol{P}_{s}$ are deactivated. A octree-based region growing (Vo et al., 2015) is employed to segment the voxel $v_{i}$, in which $p_{i} \in \boldsymbol{P}_{s}$ within the voxel $v_{i}$ is no longer consideration. To improve efficiency, only points $\boldsymbol{P}_{r p}$ close to the top and bottom of the shell wall are respectively used as input data set for extracting the roof $\left(\boldsymbol{P}_{r}\right)$ and floor $\left(\boldsymbol{P}_{f}\right)$, which is the largest segment (Step 3.1 and 3.2) (Figure 2j).

In Part 2, to create a 3D model of the shell wall, a point cloud $\boldsymbol{P}_{s}$ is linearly sliced along a cylinder direction. At each cross-section, the points within the buffer $(s b)$ are retrieved and a least square method is employed to fit a circle through these points (Figure 3a). Next, the sweeping technique is employed to generate the 3D model based on a set of the fitting circles and known thickness of the courses (Figure 3b). This methodology is used because: (1) to avoid high cost to fit the cylinder of the shell wall, and (2) to give a better shape of the shell wall when the shell wall is subjected to deformation. Notably, as thickness of the shell wall cannot automatically identify from laser scanning point cloud in this study, those values obtained from exiting documents (e.g., design documents) are used as input data. Similarly, this procedure is also applied to the points $\boldsymbol{P}_{r}$ of the roof and $\boldsymbol{P}_{f}$ of the floor to create their 3D models, in which the cross-section is created along the radial direction.

Next, to analyse deformation of the shell wall, best fit vertical deformation and roundness analysis according to requirements of
API (American Petroleum Institute, 2014) are computed using Equation 5.

$$
E_{\text {bestfit } / \text { roundness }}=d\left(p_{i}, C_{v}\right)=p_{i .} r-r_{c}
$$

where $\quad C_{v}\left(c_{c}, r_{c}, t_{c}\right)=$ the cylinder parameters consisting of a center $c_{c}$, a radius $\mathrm{r}_{c}$, and an axis direction $t_{c}$

$p_{i .} r=\mathrm{a}$ distance from the point $p_{i}$ to the cylinder axis

For the best fit vertical deformation analysis, as the shell wall is assumed as the perfect vertical cylinder, $c_{c}$ and $r_{c}$ are derived from the fitting circle based on a projection of $\boldsymbol{P}_{s}$ onto the xy plane, and $t_{c}=[0,0,1]$. For roundness, the similar procedure in Step 2.1 is used to determine the cylinder parameters. Figure 4 shows deformation from best fit vertical and roundness analyses.

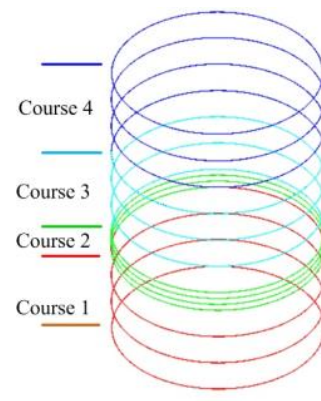

a)

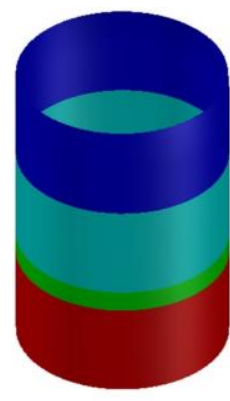

b)
Figure 3. Illustration of creating a solid model of a shell wall a) Fitting circle at each cross-section, b) a 3D solid model

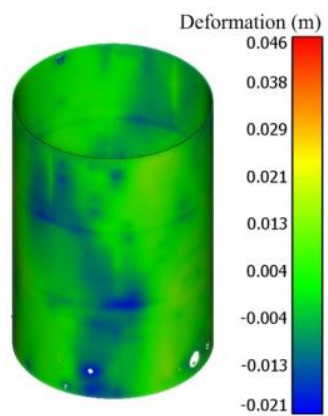

a) Best fit vertical analysis

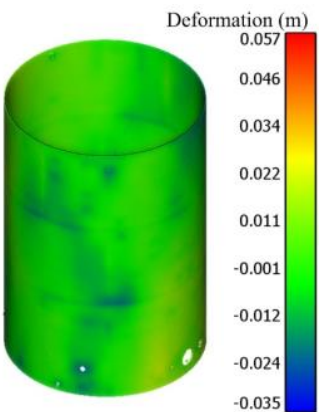

b) Roundness analysis
Figure 4. Deformation of a shell wall

Additionally, the 3D geometric model is also directly imported to FEA software to analyse behaviour of the tanks under different load scenarios to determine behaviour.

\section{EXPERIMENTAL TEST, RESULTS AND DISCUSSIONS}

To demonstrate the proposed framework, a storage tank with a diameter of $16.4592 \mathrm{~m}$ and height of $11.44 \mathrm{~m}$, located at British Columbia, Canada is selected as a case study. The tank was scanned from an interior by using Leica Scanstation P40 (Leica Geosystems, 2021). After registration, the point clouds were down-sampled to a sampling step of $5 \mathrm{~mm}$ and 22.9 million points were exported for next steps (Figure 5a).

To extract components (a shell wall, roof and floor) of a storage tank, following input parameters were: (1) for generating a voxel grid: a voxel size $v s 0=0.2 \mathrm{~m}$ and a predefined minimum number 
of points vmin_pts $=10 ;(2)$ for classifying voxels as vertical or horizontal: an angle threshold $\alpha_{\text {th }}=45$ degrees; (3) for estimating cylinder parameters using RANSAC-based voxel (Truong-Hong et al., 2020): the number of voxels to estimate the model $\mathrm{v}_{\text {model }}=$ 500 , a tolerance to identify inlier voxels $\varepsilon \max =0.01 \mathrm{~m}$, and the angle criterion ( $\alpha_{n}=5$ degrees) for a deviation between the normal vector of a voxel and normal of the cylinder. Moreover, for filtering outlier points of the shell wall and obtain points of the shell located within $v_{n s j}$ (Step 2), the bandwidth $(b w)$ of $0.01 \mathrm{~m}$ is used for generating KDE. Next, for input parameters for octreebased region growing (Vo et al., 2015) are respectively an angle, distance, and residual threshold $\alpha_{v}=5$ degrees, $d_{v}=0.01 \mathrm{~m}$, and $r_{v}=0.01 \mathrm{~m}$. The proposed method shows that all desired components (a shell wall, roof, and floor) of a tank are extracted successfully in a term of object (Figure $5 b$ and $5 c$ ).

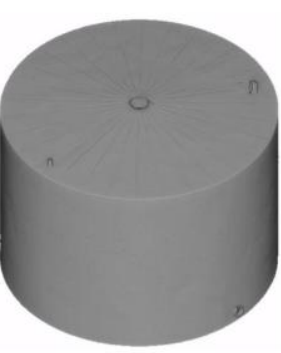

a)

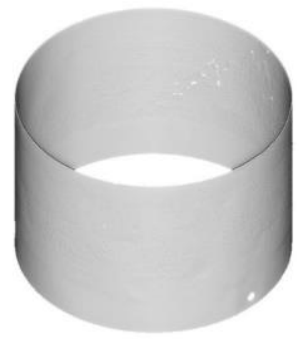

b)

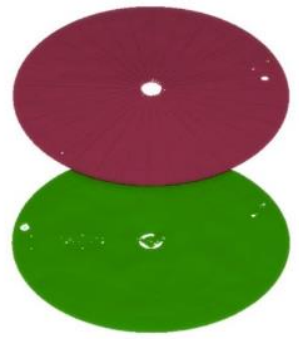

c)

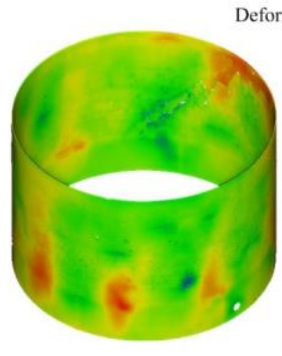

d)

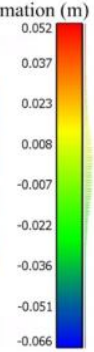

Figure 5. Extracting components of a tank and estimating deformation of a shell wall on best fit vertical analysis and roundness

To assess deformation of the shell wall, the point cloud $\left(\boldsymbol{P}_{s}\right)$ was used to estimate the cylinder parameters by using the procedure proposed by Truong-Hong et al. (2020), in which the points were used instead of the voxels as in this proposed method. Parameters of the cylinder are (1) for the best vertical deformation $c_{c}=[-$ $5.878,2.637,0.000], r_{c}=8.220, t_{c}=[0,0,1]$, and for roundness $c_{c}=[-5.878,2.639,5.473], r_{c}=8.220, t_{c}=[0.002,0.001,1.000]$. Resulting radius of the shell wall for both analyses are nearly the same and differ from the radius from design documents no more than $9.6 \mathrm{~mm}(8.2296 \mathrm{~m}$ vs. $8.220 \mathrm{~m})$. The difference can cause by either errors due to data acquisition and point cloud processing or damage of the shell wall or both. Moreover, based on the cylinder parameters, deformations of the shell wall at each data point were shown in Figure 5d and 5e. The shell wall was subjected to deformation, in which the minimum and maximum deformation were a range from $-66.0 \mathrm{~mm}$ to $52.0 \mathrm{~mm}$ for the best fit vertical analysis, and from $-66.0 \mathrm{~mm}$ to $47.0 \mathrm{~mm}$ for the roundness. Moreover, the roundness analysis shows that the shell wall was inclined 0.14 degree compared to the vertical direction $\left(t_{c}=[0.002,0.001,1.000] v s . n_{z}=[0.0,0.0,1.0]\right)$.

Additionally, the point clouds of the shell wall and floor were used to create a 3D model of the tank to demonstrate ability to use a point cloud for finite element analysis. As thickness of the shell wall varies along a vertical direction but is a constant for each course, the point cloud $\left(\boldsymbol{P}_{s}\right)$ of the shell wall were sliced along the cylinder direction. In this study, each course was divided into 4 cross-sections with an equal interval. Notably, as thickness of the shell wall and bounds of courses of the shell wall cannot automatically identify from laser scanning point cloud in this study, those values obtained from exiting documents (e.g., design drawings) are used as input data (Table 1). At each crosssection, the buffer $s b=0.01 \mathrm{~m}$ (two times of a sampling step) were used to extract the points of the cross-section, and then a fitting circle was created (Figure 6a and Table 2). Subsequently, the sweeping technique was employed to create a 3D solid model of the shell wall. Similarly, the cross-sections along a radial direction of the floor were created, in which the interval space of $1.0 \mathrm{~m}$ was used, and then the solid model of the floor was created, in which the thickness of $7.938 \mathrm{~mm}$ was used. Resulting solid models of the shell wall and floor were shown in Figure $6 \mathrm{~b}$.

\begin{tabular}{|c|c|c|c|c|c|c|}
\hline Course No. & 1 & 2 & 3 & 4 & 5 & 6 \\
\hline Height (m) & 2.457 & 2.235 & 2.235 & 2.235 & 2.127 & 0.102 \\
Shell thickness (mm) & 7.938 & 6.350 & 6.350 & 6.350 & 6.350 & 6.350 \\
\hline
\end{tabular}

Table 1. Geometric information of courses of a shell wall

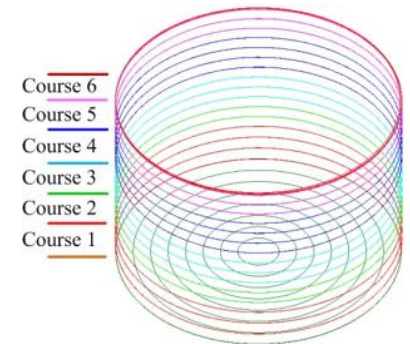

a)

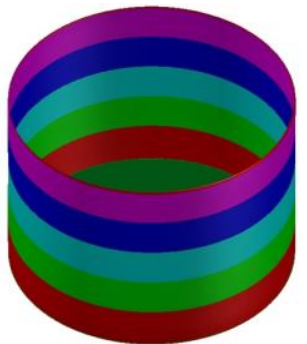

b)
Figure 6. Create a solid model of a tank (a shell wall and floor); a) fitting circles of cross-sections; b) 3D solid model

A circle of each cross-section of the shell wall was fitted from a point cloud of the section with an average of a root mean quare error of $12.5 \mathrm{~mm}$ (minimum and maximum RMSE about $6.0 \mathrm{~mm}$ and 16.mm) (Table 3). This showed that the cross-section of the shell wall is subjected to minor damage and low proportion of outlier points within a sub-dataset of the cross-section. Moreover, comparing to the radius derived from design documents, radii of fitting circles differ from ones of the design document no more than $51.6 \mathrm{~mm}$ (an average difference of $14.0 \mathrm{~mm}$ ), while RMSE is about $18.2 \mathrm{~mm}$.

The solid model of the tank was subsequently imported into to ANSYS software (Ansys, 2021a). A 3D element SOLID185 having 8 nodes associated with 3 degrees of freedom, and isotropic behaviour was used to model the shell wall and floor (Ansys, 2021b). The FEM of the tank consists of 49,943 nodes and 38,047 elements. The tank components (a shell wall, floor, 
and roof) were made from steel with mechanical properties including Young's Modulus of 210GPa and Poisson ration $\mathrm{v}=$ 0.3 . Additionally, the tank floor was seat on gravel with Young's Modulus of $20 \mathrm{MPa}$. As the goal of this analysis is to demonstrate ability of using the solid model generating directly from point cloud for FEA, the tank is assumed that crude oil filled a full tank. As such, the tank is subjected to hydraulic pressure defined the mass density $\left(\rho_{\text {crudeoil }}=875.5 \mathrm{~kg} / \mathrm{m}^{3}\right)$ and the level of crude from the shell wall top, and the boundary condition between the floor and foundation was modelled as elastic support. Results of total deformation, equivalent stress and strain were shown in Figure 7, in which maximum total displacements, equivalent stress and strain are respectively $5.05 \mathrm{~mm}, 114 \mathrm{MPa}$ and $1.6 \times 10^{-3} \mathrm{~m} / \mathrm{m}$.

\begin{tabular}{|c|c|c|c|c|c|c|c|c|c|}
\hline \multicolumn{4}{|c|}{ Fit circle } & \multirow{2}{*}{$\begin{array}{l}\text { RMSE } \\
(\mathrm{m})\end{array}$} & \multicolumn{4}{|c|}{ Fit circle } & \multirow{2}{*}{$\begin{array}{l}\text { RMSE } \\
\text { (m) }\end{array}$} \\
\hline $\mathrm{x}_{\mathrm{c}}(\mathrm{m})$ & $\mathrm{y}_{\mathrm{c}}(\mathrm{m})$ & $\mathrm{z}_{\mathrm{c}}(\mathrm{m})$ & $\mathrm{r}_{\mathrm{c}}(\mathrm{m})$ & & $\mathrm{x}_{\mathrm{c}}(\mathrm{m})$ & $\mathrm{y}_{\mathrm{c}}(\mathrm{m})$ & $\mathrm{z}_{\mathrm{c}}(\mathrm{m})$ & $\mathrm{r}_{\mathrm{c}}(\mathrm{m})$ & \\
\hline-5.874 & 2.647 & -0.116 & 8.189 & 0.006 & -5.874 & 2.639 & 7.557 & 8.218 & 0.014 \\
\hline-5.887 & 2.634 & 0.703 & 8.219 & 0.008 & -5.872 & 2.639 & 8.302 & 8.217 & 0.014 \\
\hline-5.887 & 2.635 & 1.522 & 8.218 & 0.012 & -5.869 & 2.641 & 9.047 & 8.219 & 0.014 \\
\hline-5.886 & 2.635 & 2.341 & 8.220 & 0.016 & -5.866 & 2.639 & 9.756 & 8.221 & 0.011 \\
\hline-5.885 & 2.635 & 3.086 & 8.224 & 0.015 & -5.864 & 2.641 & 10.465 & 8.227 & 0.011 \\
\hline-5.886 & 2.636 & 3.831 & 8.220 & 0.015 & -5.861 & 2.641 & 11.174 & 8.220 & 0.011 \\
\hline-5.883 & 2.637 & 4.576 & 8.215 & 0.016 & -5.861 & 2.641 & 11.234 & 8.224 & 0.011 \\
\hline-5.881 & 2.638 & 5.322 & 8.217 & 0.014 & -5.866 & 2.640 & 11.293 & 8.221 & 0.011 \\
\hline-5.879 & 2.638 & 6.067 & 8.216 & 0.014 & -5.909 & 2.663 & 11.352 & 8.178 & 0.010 \\
\hline-5.874 & 2.638 & 6.812 & 8.214 & 0.015 & & & & & \\
\hline
\end{tabular}

Table 2. Fitting circles along a vertical direction of a shell wall

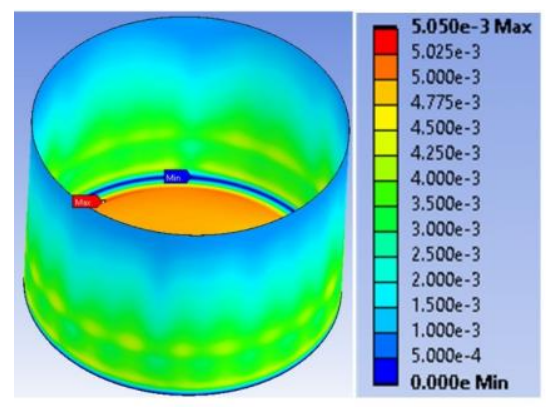

a) Total deformation (unit in $\mathrm{m}$ )

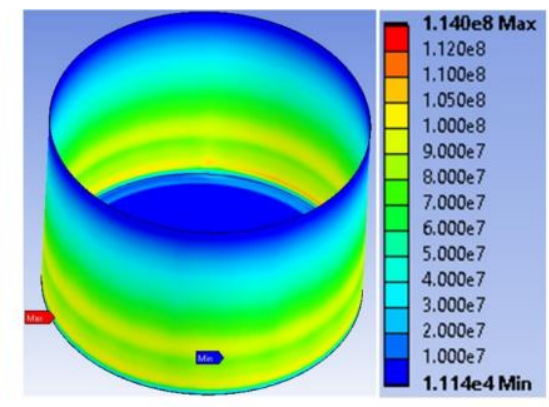

b) Equivalent stress (unit in $\mathrm{Pa}$ )

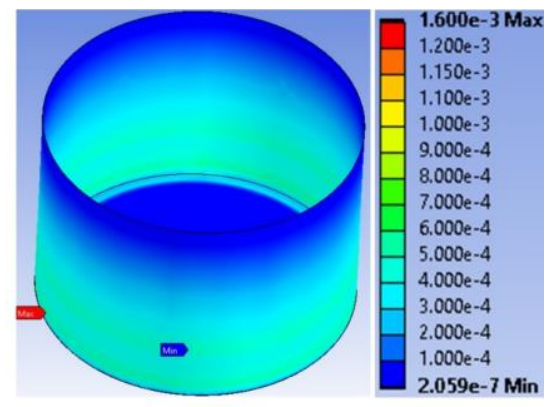

c) Equivalent stress (unit in $\mathrm{m} / \mathrm{m}$ )

Figure 7. Finite element results of an oil storage tank

\section{CONCLUSIONS}

This paper proposes a digital twin framework for oil storage tanks, in which the geometric model of the tank was created from TLS point clouds. The digital twin consists of two main parts: Part 1: extract point clouds of components of the tank, and Part 2: simulate and visualize information of the tank. This study focuses on (1) extracting a shell wall, roof, and floor, (2) analysing deformation of the shell wall, and (3) demonstrate behavior of the tank via finite element analysis, in which the solid model of the tank was automatically created from its point clouds.

To extract the point cloud of the shell wall, the proposed method called RANSAC-based voxel method developed by Truong-hong et al. (2020), originally developed to extract data points of the shell wall from the data set capturing from an exterior side of the tank, was refined. This study introduced a methodology to remove outlier points using a valley-peak-valley pattern of KDE, which allows to improve quality of extraction of shell wall. Moreover, octree-based region growing was employed to extract the roof and floor. The methodology to extract components in sequence order can reduce the complexity of the data set, and the methods were based on the voxels created from sub-sets allowing to performance of the proposed method.

The proposed framework successfully extracted point clouds of the tank components, which are subsequently used to measure deformation, and automatically create a 3D model. Best fit vertical and roundness analyses used to assess deformation of the shell wall according to API requirements were demonstrated. Roundness analysis showed that the shell wall was subjected to deformation with maximum inward and outward deformation of $66.0 \mathrm{~mm}$ and $47.0 \mathrm{~mm}$ respectively, and the shell wall was not perfectly vertical as a design stage, in which a current inclined angle of the shell wall is 0.14 degree. Moreover, the deformation can explain a difference of a cylinder radius created from a point cloud of the shell wall and design documents about $9.6 \mathrm{~mm}$. A radius of a fitting circle based on point cloud of each crosssection of the shell wall is average $14.0 \mathrm{~mm}$ differing from the radius of the designed shell wall. Finally, finite element analysis with full filled crude oil demonstrated ability to use the solid model from the point cloud to simulate behavior of the tank. 
The success of this study can offer an alternative solution to assess deformation damage of an oil tank and create a 3D solid model for simulating behaviour of the tank in automated manner. The proposed method can be integrated into a digital twin platform allowing to monitoring status of the tank nearly realtime. However, other components (e.g., columns, girders, and rafters) of the tank must be extracted and assessed. Moreover, the high accuracy of behavior of the tank can be achieved if all components are included in the FE model. In future work, the methodologies for storing different data types such as point clouds, geometric models and resulting analyses and retrieving them for visualisation and user interaction will be investigated. Moreover, impact of point cloud accuracy to results of deformation measurement and of simulation will be evaluated.

\section{REFERENCES}

American Petroleum Institute 2014. API 650: Tank Inspection, Repair, Alteration, and Reconstruction, Washington, DC, API.

Ansys 2021a. Ansys ${ }^{\circledR}$ Academic Research Mechanical, Release 19.2 .

Ansys 2021b. Ansys ${ }^{\circledR}$ Academic Research Mechanical, Release 19.2, Help System, Element Reference, ANSYS, Inc.

Hoppe, H., DeRose, T., Duchamp, T., McDonald, J., Stuetzle, W., 1992. Surface Reconstruction from Unorganized Points. ACM SIGGRAPH 1992, Jul 26-31, 1992, Chicago, USA. 71-78.

Huadong, H., Xianlei, C., Haolei, S., Xuemin, L., Pengju, Y., 2017. The Automatic Measurement System of Large Vertical Storage Tank Volume based on 3D Laser Scanning Principle. 2017 13th IEEE International Conference on Electronic Measurement \& Instruments (ICEMI), 20-22 Oct. 2017. 211-216. 10.1109/ICEMI.2017.8265768

Kassotakis, N., Sarhosis, V., Riveiro, B., Conde, B., D'Altri, A. M., Mills, J., Milani, G., de Miranda, S., Castellazzi, G., 2020. Three-dimensional Discrete Element Modelling of Rubble Masonry Structures from Dense Point Clouds. Automation in Construction, 119, 103365.

doi.org/10.1016/j.autcon.2020.103365

Leica Geosystems. 2021. Leica ScanStation P40 [Online]. Leica Geosystems. Available: https://leica-

geosystems.com/products/laser-scanners/scanners/leica-

scanstation-p40--p30 [Accessed 01/15 2020].

Pukanská, K., Gajdošík, J., Marcian, M., Bartoš, K., 2014. Determination of Deformations of a High-Capacity Tank Using the Technology of Terrestrial Laser Scanning. American International Journal of Contemporary Research, 4, 38-45. 10.30845/aijcr

Truong-Hong, L., Laefer, D., 2013. Validating Computational Models from Laser Scanning Data for Historic Facades. Journal of Testing and Evaluation, 41, 481-496. 10.1520/JTE20120243

Truong-Hong, L., Lindenbergh, R., 2019. Measuring Deformation of Bridge Structures Using Laser Scanning Data. 4th Joint International Symposium on Deformation Monitoring (JISDM), May 15-17, 2019, Athens, Greece. p.7.

Truong-Hong, L., Lindenbergh, R., 2021. Extracting Bridge Components from a Laser Scanning Point Cloud. In: Toledo
Santos, E. \& Scheer, S., eds. Proceedings of the 18th International Conference on Computing in Civil and Building Engineering, 2021, Cham. Springer International Publishing, 721-739.

Truong-Hong, L., Lindenbergh, R., Fisk, P., 2020. Storage Tank Inspection Based Laser Scanning. In: Reddy, J. N., Wang, C. M., Luong, V. H. \& Le, A. T., eds. ICSCEA 2019, 2020, Singapore. Springer Singapore, 987-996.

Truong-Hong, L., Lindenbergh, R., Nguyen, T. A., 2021. Structural Assessment Using Terrestrial Laser Scanning Point Clouds. International Journal of Building Pathology and Adaptation, 10.1108/IJBPA-04-2021-0051

Vo, A.-V., Truong-Hong, L., Laefer, D. F., Bertolotto, M., 2015. Octree-based Region Growing for Point Cloud Segmentation. ISPRS J Photogramm Remote Sens, 104, 88-100.

Wu, Z., Hou, L., Wu, S., Wu, X., Liu, F., 2020. The Time-tofailure Assessment of Large Crude Oil Storage Tank Exposed to Pool Fire. Fire Safety Journal, 117, 103192. https://doi.org/10.1016/j.firesaf.2020.103192 